\title{
Prostate cancer in young men represents a distinct clinical phenotype: gene expression signature to predict early metastases
}

\author{
Yuan C. Ding ${ }^{1}$, Huiqing Wu ${ }^{2}$, Elai Davicioni ${ }^{3}$, R. Jeffrey Karnes ${ }^{4}$, Eric A. Klein ${ }^{5}$, Robert B. Den ${ }^{6}$, Linda \\ Steele ${ }^{1}$, Susan L. Neuhausen ${ }^{1}$ \\ 'Department of Population Sciences, Beckman Research Institute of City of Hope, Duarte, California, CA 91010, USA. \\ 2Department of Pathology, City of Hope Medical Center, Duarte, California, CA 91010, USA. \\ ${ }^{3}$ GenomeDX Biosciences, Vancouver, British Columbia V6B 1B8, Canada. \\ ${ }^{4}$ Department of Urology, Mayo Clinic, Rochester, Minnesota, MN 55905, USA. \\ ${ }^{5}$ Glickman Urological and Kidney Institute, Cleveland Clinic, Ohio, OH 44195, USA. \\ ${ }^{6}$ Department of Radiation Oncology, Thomas Jefferson University, Philadelphia, Pennsylvania, PA 19044, USA.
}

Correspondence to: Prof. Susan L. Neuhausen, Department of Population Sciences, Beckman Research Institute of City of Hope, 1500 E Duarte Road, Duarte, CA 91010, USA. E-mail: sneuhausen@coh.org

\begin{abstract}
How to cite this article: Ding YC, Wu H, Davicioni E, Karnes RJ, Klein EA, Den RB, Steele L, Neuhausen SL. Prostate cancer in young men represents a distinct clinical phenotype: gene expression signature to predict early metastases. J Trans/ Genet Genom 2021;5:50-61. http://dx.doi.org/10.20517/jtgg.2021.01
\end{abstract}

Received: 3 Jan 2021 First Decision: 27 Jan 2021 Revised: 2 Feb 2021 Accepted: 18 Feb 2021 Available online: 9 Mar 2021

Academic Editor: Sanjay Gupta Copy Editor: Yue-Yue Zhang Production Editor: Yue-Yue Zhang

\begin{abstract}
Aim: Several genomic signatures are available to predict Prostate Cancer ( $\mathrm{CaP}$ ) outcomes based on gene expression in prostate tissue. However, no signature was tailored to predict aggressive $\mathrm{CaP}$ in younger men. We attempted to develop a gene signature to predict the development of metastatic $\mathrm{CaP}$ in young men.
\end{abstract}

Methods: We measured genome-wide gene expression for 119 tumor and matched benign tissues from prostatectomies of men diagnosed at $\leq 50$ years and $>70$ years and identified age-related differentially expressed genes (DEGs) for tissue type and Gleason score. Age-related DEGs were selected using the improved Prediction Analysis of Microarray method (iPAM) to construct and validate a classifier to predict metastasis using gene expression data from 1,232 prostatectomies. Accuracy in predicting early metastasis was quantified by the area under the curve (AUC) of receiver operating characteristic (ROC), and abundance of immune cells in the tissue microenvironment was estimated using gene expression data.

Results: Thirty-six age-related DEGs were selected for the iPAM classifier. The AUC of five-year survival ROC for the iPAM classifier was 0.87 ( $95 \% \mathrm{Cl}: 0.78-0.94)$ in young ( $\leq 55$ years), 0.82 ( $95 \% \mathrm{Cl}: 0.76-0.88$ ) in middle-

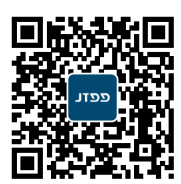


aged (56-70 years), and 0.69 ( $95 \% \mathrm{Cl}: 0.55-0.69)$ in old (> 70 years) patients. Metastasis-associated immune responses in the tumor microenvironment were more pronounced in young and middle-aged patients than in old ones, potentially explaining the difference in accuracy of prediction among the groups.

Conclusion: We developed a genomic classifier with high precision to predict early metastasis for younger $\mathrm{CaP}$ patients and identified age-related differences in immune response to metastasis development.

Keywords: Differentially expressed gene, immune cell enrichment, metastasis, prostate cancer, tissue microenvironment, age, prediction, patient stratification, clinical phenotype

\section{INTRODUCTION}

Prostate cancer $(\mathrm{CaP})$ is primarily a disease of older men; only $9.2 \%$ of men develop CaP under age 55 years ${ }^{[1]}$. Although the overall incidence of $\mathrm{CaP}$ is decreasing in the United States ${ }^{[1]}$, the incidence is increasing in younger ( $\leq 55$ years) men compared to older men $(>70 \text { years })^{[2-4]}$. Due to a longer life expectancy, younger men with localized $\mathrm{CaP}$ are more likely to receive radical prostatectomy (RP) treatment than older $\operatorname{men}^{[5,6]}$. A recent long-term follow-up study demonstrated that only those patients harboring lethal $\mathrm{CaP}$ and having a long-life expectancy benefited from RP treatment ${ }^{[7]}$. Over the past decade, several genomic signatures have been developed to predict $\mathrm{CaP}$ outcomes based on gene expression in prostatectomy or biopsy tissues ${ }^{[8]}$. However, no prognostic signature was tailored to predict aggressive $\mathrm{CaP}$ in men younger than age 55 years. Converging data from clinical and molecular genetic studies provide strong evidence that $\mathrm{CaP}$ in young men represents a distinct clinical phenotype with underlying biological differences compared to older men ${ }^{[9-13]}$. We hypothesized that age-related differences in tumor biology have implications for prognosis of early-onset CaPs. To test this hypothesis, we selected tumor and matched benign prostatic tissue samples from men diagnosed with $\mathrm{CaP}$ at younger ( $\leq 50$ years) and older (71-75 years) ages with low (6), intermediate (7), and high (8-10) Gleason scores. We identified age-related differentially expressed genes (DEGs) by comparing sample type (tumor versus matched benign) and Gleason scores (low vs. high). Then we developed a genomic classifier using gene expression of age-related DEGs and tested the classifier for accurate identification of young patients with aggressive CaP as defined by metastasis within five years of RP.

\section{METHODS}

\section{Patient characteristics, mRNA profiling, and identification of age-related DEGs}

This study was approved by the City of Hope (COH) Institutional Review Board (IRB07244). Patients with $\mathrm{CaP}$ and treated with RP between 1998 and 2013 at COH National Medical Center were selected based on age at diagnosis and tissue availability [Table 1]. A total of 61 men diagnosed between the ages of 71 and 75 years (old) and 58 men diagnosed between the ages of 38 and 50 years (young) were used to identify agerelated DEGs for developing a gene expression classifier to predict metastasis following RP. Older cases were matched to younger cases for cancer stage and Gleason score. Tissue processing and mRNA profiling were performed as described ${ }^{[9]}$. Follow-up data were abstracted from medical records and the $\mathrm{COH}$ cancer registry. Age-related DEGs were identified from expression data using a mixed linear model implemented in limma $\mathrm{R}^{[14]}$ [Supplementary methods].

\section{iPAM classifier development and validation}

Gene expression data (46,050 genes and 1,232 patients from RP) from the Decipher Genomic Resource Information Database (GRID, Decipher Biosciences, San Diego, CA) [Supplementary Table 1] were used to develop and validate a new genomic classifier. The study design is shown in Figure 1. Gene expression data for the age-related DEGs were extracted from the Mayo Clinic (MC I) discovery cohort ${ }^{[15]}$. A two-sample 
Table 1. Clinical and demographic characteristics of 119 City of Hope patients

\begin{tabular}{llll}
\hline & \multicolumn{1}{c}{ Total } & $\begin{array}{c}\text { Old } \\
(\mathbf{7 1 - 7 5} \text { years) }\end{array}$ & $\begin{array}{c}\text { Young } \\
(\mathbf{3 8 - 5 0} \text { years) }\end{array}$ \\
\hline Total patients & 119 & 61 & 58 \\
Metastatic patients & 11 & 4 & 7 \\
Mean follow-up (months) & & 65.3 & 65.8 \\
Pathology stage & & & \\
2a & $12(0.10)$ & $5(0.08)$ & $7(0.12)$ \\
2b & $2(0.02)$ & $0(0)$ & $2(0.03)$ \\
2c & $77(0.65)$ & $41(0.67)$ & $36(0.62)$ \\
3a & $19(0.16)$ & $10(0.16)$ & $9(0.16)$ \\
3b & $9(0.07)$ & $5(0.08)$ & $4(0.07)$ \\
Gleason score & & & \\
6 & $37(0.31)$ & $18(0.30)$ & $19(0.33)$ \\
$7^{\star}$ & $49(0.41)$ & $25(0.41)$ & $24(0.41)$ \\
8 or 9 & $33(0.28)$ & $18(0.29)$ & $15(0.26)$ \\
PrePSA^ (ng/mL) & & & \\
$\leq 10.0$ & $100(0.84)$ & $53(0.87)$ & $47(0.81)$ \\
>10.0 & $19(0.16)$ & $8(0.13)$ & $11(0.19)$ \\
Race & & & \\
Caucasian & $110(0.92)$ & $57(0.93)$ & $53(0.91)$ \\
Asian & $2(0.02)$ & $2(0.03)$ & 0 \\
African American & $6(0.05)$ & $2(0.03)$ & $4(0.07)$ \\
Native American & $1(0.01)$ & 0 & $1(0.02)$ \\
\hline
\end{tabular}

*data for Gleason 7 patients were reported previously; ^PSA level before surgery; PSA: Prostate specific antigen.

A total of 5156 differentially expressed genes (DEGs) in relation to age, sample type, and Gleason score identified in tumor and matched benign prostatic tissue samples from City of Hope.

\section{DEGs}

Applied to gene expression data of 545 Mayo-Clinic samples obtained from Decipher Biosciences to identify genes differentially expressed between metastatic after prostatectomy and non-metastatic patients

\section{DEGs}

Used iPAM program to select set of genes that predicted metastasis in the training set of 362 (randomly assigned from total set of 545)

$$
36 \text { DEGs }
$$

Tested the 36 DEGs using iPAM program for metastasis prediction in the test set of 183 samples (remainder of the total set of 545 samples)

$$
36 \text { DEGs }
$$

Assembled the 36 DEGs into an iPAM classifier by fitting a logistic regression model on the 362 samples in the training set

$$
\text { iPAM classifier (36 DEGs) }
$$

Predicted metastatic status for four independent validation data sets from Decipher Biosciences

Figure 1. Study design for developing the iPAM classifier.

$t$-test selected DEGs between patients with and without metastasis. After DEG determination, patients were randomly assigned into training $(n=362)$ and test $(n=183)$ datasets. An improved Prediction Analysis of Microarray (iPAM) method ${ }^{[16-18]}$ removed DEGs irrelevant to metastasis prediction based on minimizing the 10-fold cross-validated error rate using the Adaptive Hierarchically Penalized Nearest Shrunken Centroid algorithm ${ }^{[17]}$. These iPAM-selected DEGs were assembled into an iPAM classifier by fitting a logistic regression model on the training set. 
Table 2. The number of DEGs in relation to age, sample type, and Gleason score

\begin{tabular}{lll}
\hline${ }^{*} \mathbf{D E G s}$ (|fold| > 1.5 and FDR < 0.05) & $\begin{array}{l}\text { Old patients } \\
\text { (aged 71-75) }\end{array}$ & $\begin{array}{l}\text { Young patients } \\
\text { (aged 38-50) }\end{array}$ \\
\hline Tumor versus benign tissue comparison & & \\
Patients with Gleason sum of 6 & 1250 & 1314 \\
Patients with Gleason sum of 7 & 1443 & 1485 \\
Patients with Gleason sum of 8+ & 3221 & 1923 \\
Low versus high Gleason score comparison & 1392 & 650 \\
\hline
\end{tabular}

${ }^{\star}$ A total of 5156 unique DEGs identified from 8 different comparisons; ' Low Gleason score of 6 and high Gleason score of 8+ (8 to 10). DEGs: Differentially expressed genes.

Three independent validation data sets [Supplementary Table 1] with follow-up time [the Mayo Clinic II $(\mathrm{MC} \mathrm{II})^{[19]}$, the Cleveland Clinic $(\mathrm{CC})^{[20]}$, and the Thomas Jefferson University (TJU) ${ }^{[21]}$ were used to evaluate the performance of the iPAM classifier by AUC of ROC for censored survival data ${ }^{[19]}$. The $95 \%$ confidence interval for AUC of survival ROC was constructed from 1000 bootstrap replications. Based on bimodal distribution of risk scores predicted by the iPAM classifier, two cut points were selected to categorize patients into low-, intermediate-, and high-risk groups. Kaplan-Meier estimator and a log-rank test were used to evaluate the difference in time to metastasis among the risk groups. The conventional AUC of ROC was calculated to measure prediction accuracy for the fourth validation data set from the Memorial Sloan-Kettering Cancer Center (MSKCC), which had no follow-up time but categorical metastasis status for each patient.

\section{Estimation of cell-type proportion in tissue microenvironment}

$\mathrm{xCell}^{[22]}$ was used to estimate the abundance of 34 immune cell types for each tissue sample using genomewide gene expression data. Cell-type proportion in tissue microenvironment estimated by xCell method is a rank-based enrichment score. Non-parametric analysis of variance (ANOVA) (confidence interval and p-values generated by percentile bootstrap) implemented in the "Rallfun-v35" R codes from Dr. Wilcox ${ }^{[23]}$, was used to test median differences in immune score (average abundance of immune cells) between sample groups classified by factors of sample type (tumor, benign), metastasis status (yes, no), and age group (young, middle-aged, old).

\section{RESULTS}

\section{Identification of age-related DEGs}

We previously identified genes differentially expressed between tumor and matched benign prostatic tissue samples for young men $\left(\leq 45\right.$ years) and old men $\left(71-74\right.$ years) with Gleason score $7(3+4) \mathrm{CaP}^{[9]}$. Following the same study design, we generated gene expression data for tumor and matched benign prostatic samples from young men ( $\leq 50$ years, $n=34$ ) and old men ( $71-75$ years, $n=36$ ) with CaP Gleason scores of 6 or 8-10. We identified 5,156 unique DEGs as potential candidate genes for developing the iPAM classifier [Table 2]. Dot plots of gene expression for two DEGs are shown in Figure 2A and Figure 2B as examples. Details on the 5156 DEGs are available upon request.

\section{iPAM classifier development and performance assessment}

Gene expression data for the 5156 DEGs were extracted from the MC I discovery data set. Of those DEGs, 419 were differentially expressed (false discovery rate $[$ FDR $]<0.05$ ) between patients who did and did not develop metastasis. The iPAM program ${ }^{[18]}$ selected 36 genes [Table 3] of the 419 that predicted metastatic $\mathrm{CaP}$ in the training dataset and then generated an AUC of 0.75 for the test data set. We assembled those 36 genes into an iPAM classifier by fitting a logistic regression model on the training samples, and applied the iPAM classifier to four independent validation data sets. The predicted iPAM risk scores for metastasis showed a bimodal distribution with the score range of $0-1$, where higher scores represent higher risk of 

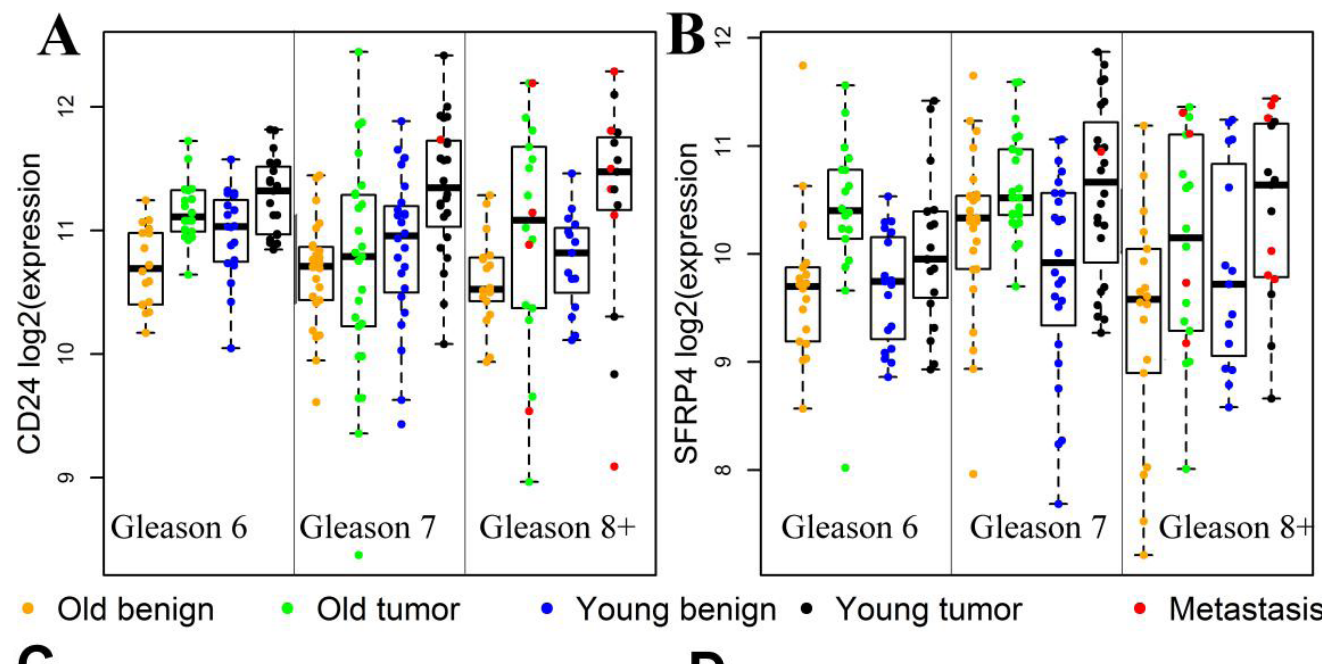

C
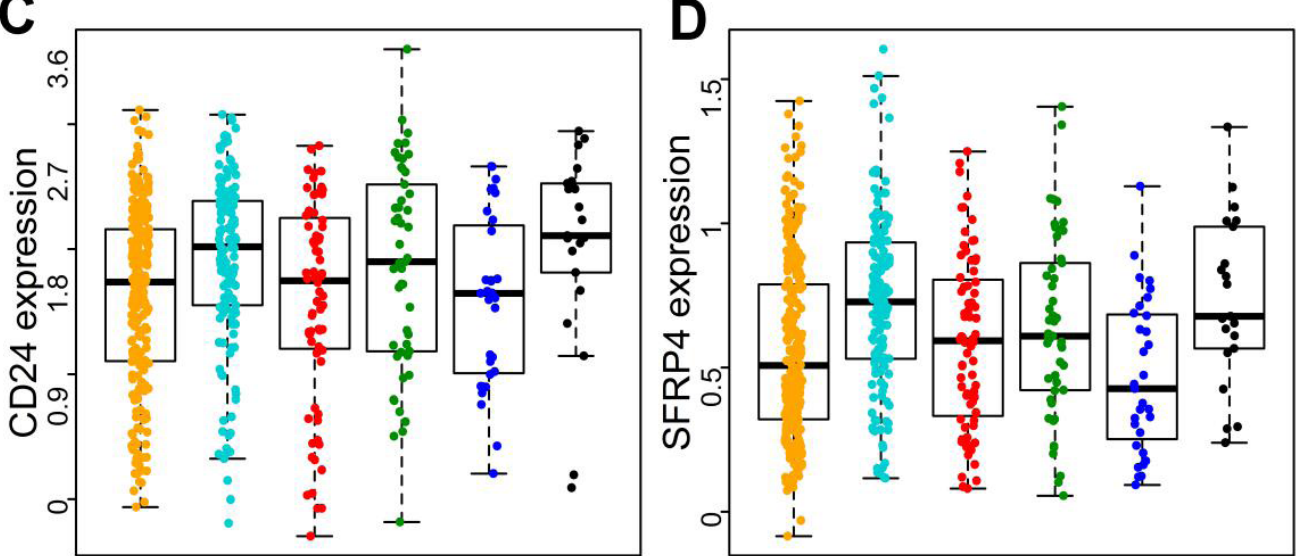

Age $56-70$, no metastasis - Age $>70$, no metastasis

- Age 56-70, metastasis

- Age $>70$, metastasis

- Age $\leq 55$, no metastasis

- Age $\leq 55$, metastasis

Figure 2. Differential gene expression for CD24 and SFRP4 between sample groups classified by patient age, sample type, or metastasis factors. CD24 and SFRP4 are 2 of the 36 genes in the iPAM classifier. A and B, Gene expression data from the 119 COH patients were used to generate the box and dot plots; there were significant tumor-vs.-matched-benign median expression differences among young patients ( $\leq 50$ years) with Gleason score of 7 for CD24 (A) and SFRP4 (B) (black vs. blue for young patients, FDR < 0.05); for CD24 (panel A), median expression level was significantly higher in tumors from young patients than in tumors from old patients with Gleason score of 7 (black vs. green, FDR < 0.05). C and D, Gene expression data for 545 patients in the Mayo discovery data set were used to generate plots; among young ( $\leq 55$ years) patients, significantly increased median expression levels for CD24 (C) and SFRP4 (D) were observed in patients with metastasis compared to patients without metastasis (black vs. blue, FDR $<0.05$ ); however, no significant median expression difference related to metastasis status among patients older than 70 years (red vs. green, $P>0.2$ ).

developing metastasis. For the three independent data sets (MC II, CC, TJU, $n=556$ ) with follow-up time from the date of RP, 75 of 556 patients (13.5\%) developed metastasis within five years after RP (early), 60 patients (10.8\%) developed metastasis more than five years after RP (late), and 421 patients had no metastases at last follow-up (mean follow-up time of 91 months). The median iPAM risk scores for the three groups of patients were $0.89,0.67$, and 0.24 , respectively. The iPAM risk scores were stratified into high-, intermediate-, and low-risk groups by two cut points of 0.4 and 0.6 determined by the distribution of risk score [Supplementary Figure 1]; the three groups showed highly significant differences in metastasisfree survival $(P<0.0001)$ [Figure $3 \mathrm{~A}$ ]. Of 75 patients who developed metastasis within five years of RP, 69 patients (92\%) were classified in either the high-risk group (58 patients) or intermediate-risk group (11 patients). The AUC of five-year survival ROC for the iPAM classifier was 0.82 (95\%CI: 0.77-0.86), outperforming the AUC [0.69 (95\%CI: 0.61-0.75)] for the clinical classifier assembled based on six clinical variables [Supplementary methods]. With a combined clinical and PAM classifier, the AUC was 0.80 (95\%CI: 0.75-0.85). 
Table 3. Function and pathway annotation of 36 genes in the iPAM classifier

\begin{tabular}{|c|c|c|c|c|c|}
\hline $\begin{array}{l}\text { Gene } \\
\text { name }\end{array}$ & $\begin{array}{l}\text { Bone-disease } \\
\text { related }\end{array}$ & $\begin{array}{l}\text { Immune } \\
\text { pathway } \\
\text { related }\end{array}$ & $\begin{array}{l}\text { Cell adhesion } \\
\text { /cell-matrix } \\
\text { related }\end{array}$ & $\begin{array}{l}\text { Cell } \\
\text { cycle }\end{array}$ & $\begin{array}{l}\text { Gene function, disease association, and pathway role (abstracted } \\
\text { from the gene card website, https://www.genecards.org/) }\end{array}$ \\
\hline ANO7 & & & Yes & & $\begin{array}{l}\text { Associated with advanced CaP, a target in CaP diagnosis and } \\
\text { immunotherapy }\end{array}$ \\
\hline ANTXR1 & & Yes & Yes & & TLR Pathway, ECM proteins, actin cytoskeleton and promotes cell spreading \\
\hline ASPN & Yes & & Yes & & $\begin{array}{l}\text { Degradation of extracellular matrix (ECM) proteoglycans, associated with } \\
\text { Osteoarthritis }\end{array}$ \\
\hline ATP5EP2 & & & & & Purine nucleotides de novo biosynthesis \\
\hline$A Z G P 1$ & & Yes & & & $\begin{array}{l}\text { Member of macroglobulin family; antigen binding; validated predictor of } \\
\text { metastatic CaP }\end{array}$ \\
\hline C7 & & Yes & & & Pathway of the innate immune system; involved in host immunity \\
\hline CCDC6 & & & & & $\begin{array}{l}\text { Structural constituent of cytoskeleton, associated with thyroid papillary } \\
\text { carcinoma }\end{array}$ \\
\hline CD24 & & Yes & & & Modulates growth and differentiation signals to granulocytes and B cells \\
\hline CDC42EP5 & & & Yes & & Induces actin filament assembly leading to cell shape changes. \\
\hline CYBA & & Yes & & & $\begin{array}{l}\text { TNFR1 Pathway and Class I MHC mediated antigen processing and } \\
\text { presentation }\end{array}$ \\
\hline DDIT4 & & Yes & & & DDIT4/mTOR axis involved in the differentiation of Th17 cells \\
\hline DPT & & & Yes & & Cell-matrix interactions and matrix assembly, mediate adhesion \\
\hline FAM13C1 & & & & & GTPase activator activity \\
\hline FBXL8 & & Yes & & & Involved in Class I MHC mediated antigen processing and presentation \\
\hline GLO1 & Yes & & & & $\begin{array}{l}\text { Risk factor for CaP progression and involved in bone formation and } \\
\text { resorption }\end{array}$ \\
\hline GLYATL1 & & & & & $\begin{array}{l}\text { Cytochrome P450 - arranged by substrate type and Conjugation of } \\
\text { carboxylic acids }\end{array}$ \\
\hline GMNN & & & & Yes & $\begin{array}{l}\text { Cell cycle regulation, increased expression plays role in colon, rectal and } \\
\text { breast cancer }\end{array}$ \\
\hline GNPTAB & Yes & & & & $\begin{array}{l}\text { Associated with mucolipidosis II and IIIA with low bone mineral density } \\
\text { (osteoporosis) }\end{array}$ \\
\hline ITGBL1 & & & Yes & & $\begin{array}{l}\text { Participates in cell adhesion as and cell-surface, cell-cell and cell-matrix } \\
\text { interaction. }\end{array}$ \\
\hline KIF21A & & & Yes & & Involved in microtubule transport, involved in cell polarity, and migration \\
\hline KRT15 & & Yes & Yes & & $\begin{array}{l}\text { Involved in the structural integrity of epithelial cells; regulating innate } \\
\text { immunity }\end{array}$ \\
\hline$\angle B H$ & & & & & Contributes to Wnt-induced tumorigenesis \\
\hline LRRN1 & & & & & Regulates Differentiation of Embryonic Stem Cells \\
\hline LTF & & Yes & & & $\begin{array}{l}\text { Anti-inflammatory activity, induce apoptosis and inhibit proliferation in } \\
\text { cancer cells. }\end{array}$ \\
\hline MTDH & Yes & & & & $\begin{array}{l}\text { Promotes lung metastasis and also has an effect on bone and brain } \\
\text { metastasis }\end{array}$ \\
\hline MYBPC1 & & & Yes & & Contributes to the stability and maintenance of sarcomeres. \\
\hline NCAPD3 & & & & Yes & Chromosome condensation in prometaphase and Cell Cycle, Mitotic \\
\hline NR4A1 & & Yes & & & Involved in Class I MHC mediated antigen processing and presentation \\
\hline PCA3 & & & & & $\begin{array}{l}\text { Sensitive to androgen-receptor activation, a molecular signature of prostate } \\
\text { cancer }\end{array}$ \\
\hline RNF39 & & & & & Plays a role in an early phase of synaptic plasticity \\
\hline SFRP4 & Yes & & & & $\begin{array}{l}\text { Modulators of Wnt signaling, associated with Osteomalacia and Pyle } \\
\text { Disease }\end{array}$ \\
\hline SLC22A3 & & & & & Disposition of small organic cations and drugs and environmental toxins \\
\hline SLC37A3 & & & & & Transporter activity and transmembrane transporter activity \\
\hline STRBP & & & & & DNA and RNA binding, plays a role in regulation of cell growth \\
\hline TOP2A & & & & Yes & $\begin{array}{l}\text { Associated with aggressive } \mathrm{CaP} \text { and drug resistance; Essential in mitosis } \\
\text { and meiosis }\end{array}$ \\
\hline$U G D H$ & & & Yes & & $\begin{array}{l}\text { Components of the ECM involved in cell migration, and cancer growth and } \\
\text { metastasis }\end{array}$ \\
\hline
\end{tabular}

${ }^{*}$ ANO7 and MYBPC1 are also in the Decipher classifier ${ }^{[15]}$; Top2A is also in the Prolaris classifier ${ }^{[27]} ; A Z G P 1$ and SFRP4 are also in the Oncotype DX classifier ${ }^{[26]}$; IPAM: Improved prediction analysis of microarray.

The AUC of survival ROC for the iPAM classifier by Gleason scores and age groups is shown in Table 4. The highest AUC of 0.87 (95\%CI: 0.78-0.94) was observed in young patients (age $\leq 55$ years); an intermediate AUC of 0.82 (95\%CI: 0.76-0.88) was observed for middle-aged patients (age 56-70 years), and the lowest AUC of 0.69 (95\%CI: 0.55-0.82) was observed in old patients (age $>70$ years) [Figure 3B]. For patients with Gleason scores of 7-10, the overall AUC was 0.80 (95\%CI: 0.74-0.85), with the highest AUC of 0.85 (95\%CI: 0.75-0.93) in the young group and the lowest AUC of 0.67 (95\% CI: 0.50-0.80) in the old group. The iPAM 

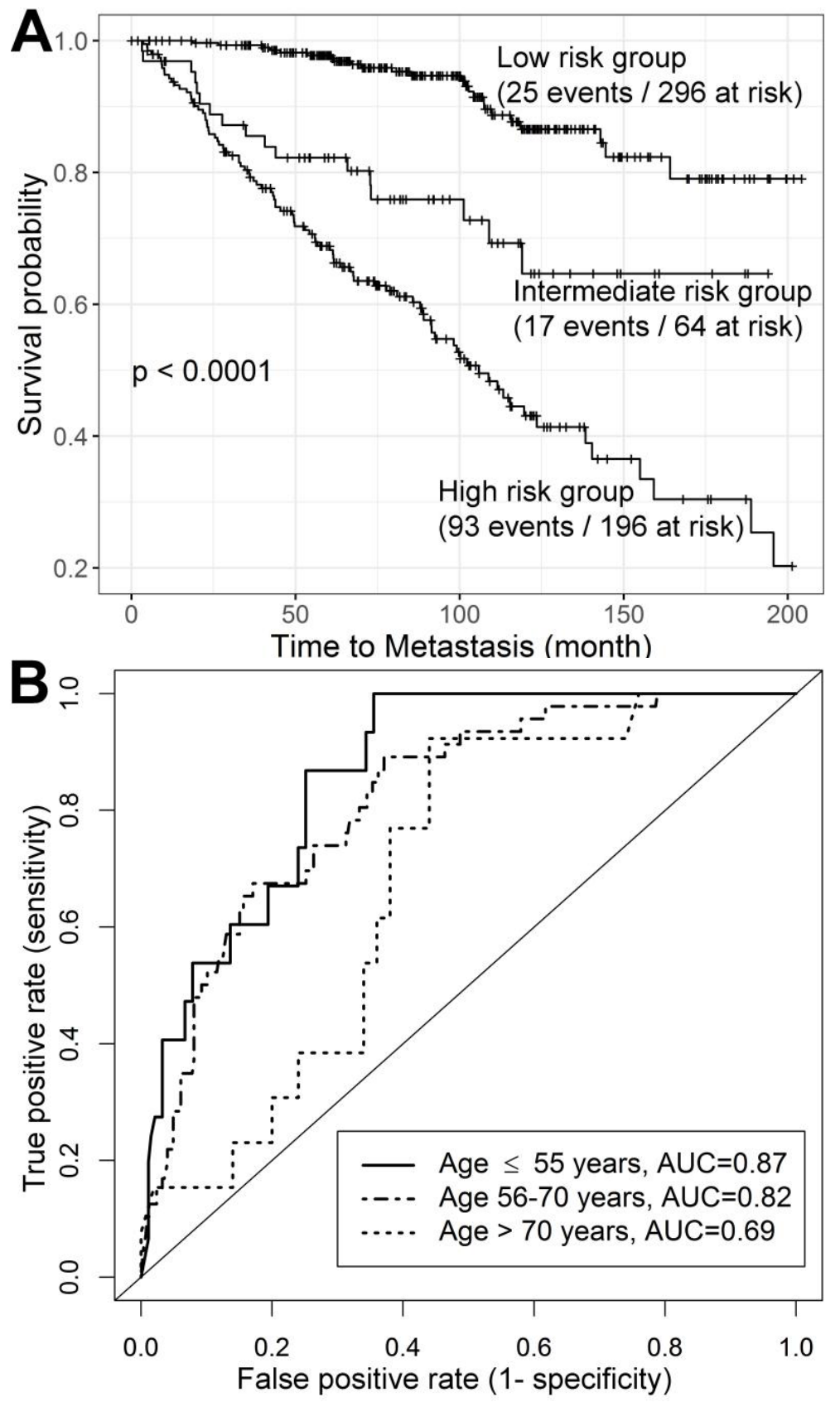

Figure 3. Performance of the iPAM classifier. Combination of patients from Mayo Clinic validation data $(n=235)$, Cleveland Clinic validation data $(n=182)$, and Thomas Jefferson University validation data $(n=139)$ were used to evaluate performance of the iPAM classifier. (A) Three risk groups [low- (iPAM risk score $<0.4$ ), intermediate- $(0.4 \leq$ iPAM risk score $\leq 0.6$ ), and high-risk (iPAM risk score $>0.6)$ group] showed highly significant differences in metastasis-free survival $(P<0.0001)$ from the Kaplan-Meier survival analysis. (B) Accuracy in predicting early metastasis (within five years of RP), quantified by AUC of ROC, was higher in young ( $\leq 55$ years) $(A \cup C=0.87$ ) and middle-aged patients ( $A \cup C=0.82)$ than in old $(>70$ years) patients $(A \cup C=0.69)$.

classifier demonstrated slightly higher prediction accuracy for patients with Gleason score of 7 compared to prediction scores for all patients.

For the MSKCC data set with no information on time of follow-up (9 patients with metastasis versus 122 without metastasis), the conventional AUC calculated with a binary metastasis status was 0.86 (95\%CI: 0.73-0.99) [Supplementary Figure 2]. 
Table 4. AUC of five-year survival ROC for validation data stratified by Gleason score and age at diagnosis

\begin{tabular}{lllll}
\hline Gleason score & \multicolumn{1}{c}{ Age 40-78 } & \multicolumn{1}{c}{ Age $\leq \mathbf{5 5}$} & \multicolumn{1}{c}{ Age 56-70 } & \multicolumn{1}{c}{ Age $>$ 70 } \\
\hline $6-10$ & $(n=421,135)$ & $(n=79,23)$ & $(n=298,93)$ & $(n=44,19)$ \\
& $0.82(0.77,0.86)$ & $0.87(0.78,0.94)$ & $0.82(0.76,0.88)$ & $0.69(0.55,0.82)$ \\
$7-10$ & $(n=357,134)$ & $(n=60,23)$ & $(n=257,92)$ & $(n=40,19)$ \\
& $0.80(0.74,0.85)$ & $0.85(0.75,0.93)$ & $0.81(0.74,0.86)$ & $0.67(0.50,0.80)$ \\
7 & $(n=252,58)$ & $(n=48,12)$ & $(n=184,41)$ & $(n=20,5)$ \\
& $0.83(0.74,0.90)$ & $0.87($ NA $)$ & $0.83(0.73,0.91)$ & $0.79(\mathrm{NA})$ \\
\hline
\end{tabular}

*The number of patients without and with metastasis, respectively; ${ }^{\wedge}$ insufficient sample of metastatic patients to calculate $95 \%$ confidence interval by the bootstrap method.

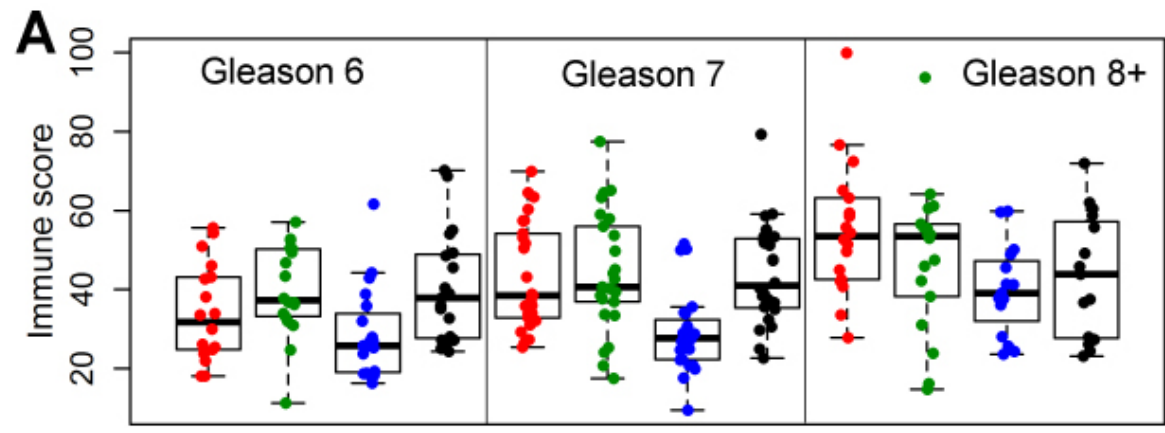

- Age $>70$, normal tissue - Age $\leq 50$, normal tissue

- Age $>70$, tumor tissue - Age $\leq 50$, tumor tissue

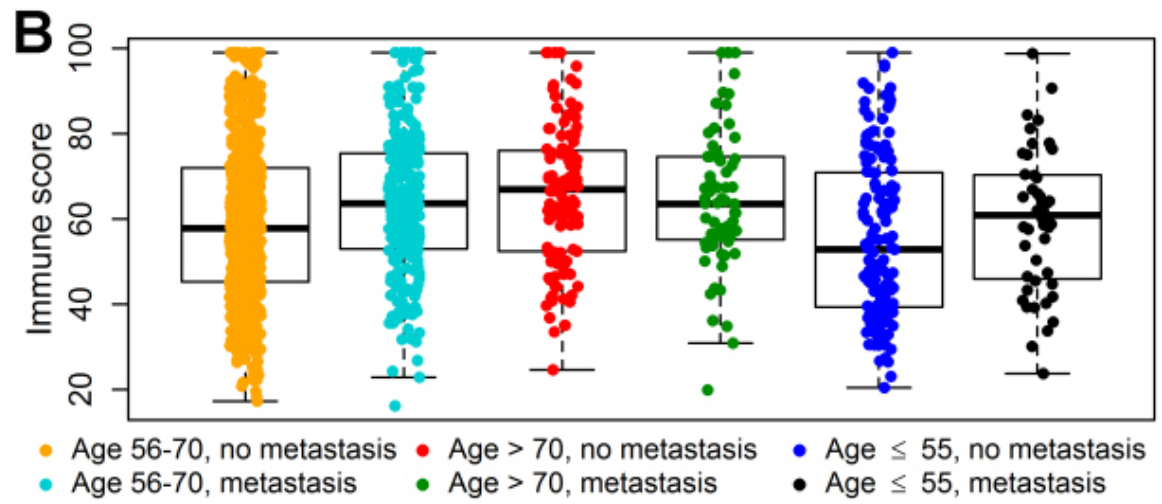

Figure 4. Differential abundance of immune cells (immune score) in tissue microenvironment between sample groups classified by patient age, sample type, or metastasis factors. (A) Immune scores for tumor and matched benign prostatic tissue samples from 119 patients in the $\mathrm{COH}$ data set were used to generate dot and box plots. Compared to matched benign prostatic tissue, tumor tissues with Gleason scores of 6 and 7 from young patients ( $\leq 50$ years) (black) showed significantly increased abundance of immune cells (blue vs. black, $P<0.01$ ), whereas old patients (> 70 years) showed non-significant tumor-versus-matched-benign difference in abundance of immune cells (red vs. green, $P>0.10$ ). (B) Immune scores for 1232 primary tumor samples from five GRID data sets were used to generate plots. Compared to tumor tissues from young patients ( $\leq 55$ years) without metastasis, tumor tissues from young patients with metastasis showed significantly (blue vs. black, $P=0.02$ ) increased abundance of immune cell types; patients with middle age (56-70 years) also showed highly significant but a smaller with-and-without- metastasis difference (cyan vs. orange, $P<0.0001$ in abundance of immune cell types than young patients ( $\leq 55$ years); old patients ( $\geq 70$ years) (green vs. red, $P=0.21$ ) had no significant difference in abundance of immune cell types related to metastasis status.

\section{Estimation of abundance of immune cell types}

The relative abundance of immune cells for tumor and matched benign prostatic tissue samples from 119 patients is displayed in Figure 4A. Compared to matched benign samples, there was a significant enrichment $(P<0.01$, Supplementary Table 2 of immune cells in tumor samples (Gleason scores 6-7) from young patients, with no significant differences in the old patients, indicating stronger tumor-induced immune responses among young patients than among old patients. For tumors with high Gleason scores (8-10), the tumor-benign difference did not reach statistical significance. 
The relative abundance of immune cells for primary tumor samples from 1,232 patients in the 5 GRID data sets is shown in Figure 4B. In young patients, there was a significantly $(P=0.028)$ greater abundance of immune cells in primary tumors from patients with metastasis compared to primary tumor samples from patients without metastasis; there were no significant metastasis-associated differences in the old patient group. In middle-aged patients, abundance of immune cells from patients with metastasis was significantly greater than that in the patients without metastasis $(P<0.0001$, Supplementary Table 3$)$. Four immune cell types demonstrated striking age-related differences in abundance of immune cell type [Supplementary Figure 3]. For the 687 patients from the four independent validation data sets (MC II, CC, TJU, and MSKCC), the predicted iPAM risk scores for metastasis were significantly associated with the immune scores (Spearman rho $=0.25, P$-value $=2.68 \times 10^{-11}$ ), indicating that gene expression for the 36 iPAM genes may capture information on immune responses in the tumor microenvironment that lead to metastasis.

\section{DISCUSSION}

Prostate-specific antigen screening has reduced death from $\mathrm{CaP}$ due to early detection while also leading to over-treatment of low-risk $\mathrm{CaP}^{[24,25]}$. Three commercially available genomic classifiers (Oncotype $\mathrm{DX}^{[26]}$, Prolaris ${ }^{[27]}$, and Decipher $\left.{ }^{[15]}\right)$ are used to predict metastatic CaP and guide initial treatment and/or postoperative intervention. However, those classifiers were not designed for predicting metastatic disease in young patients and accuracy of prediction for young men with $\mathrm{CaP}$ was not examined. This is the first study to investigate young men with $\mathrm{CaP}$ as a distinct and unique patient group in both discovery and validation of prediction signatures for early metastatic disease.

The accuracy of early metastasis prediction, measured by AUC of five-year survival ROC for the Decipher in the three validation data sets (MC II, CC, and TJU) with follow-up time from date of RP are shown in Supplementary Table 4. The AUC of five-year ROC generated from the iPAM classifier was higher than that from the Decipher classifier. If only the six clinical variables were used to predict metastasis, AUC was 0.69 for both data sets; therefore, both the Decipher and iPAM classifiers showed substantial improvement on prediction of early metastasis compared to the clinical classifier alone. Inclusion of the clinical variables into the genomic classifier did not improve prediction accuracy for both classifiers. This suggests that both genomic classifiers captured the prediction information provided by the clinical variables.

To develop the iPAM classifier, we selected DEGs associated with sample-type (tumor or benign) factor, Gleason score factor, and metastasis factor. It is known that: (1) genes differentially expressed between tumor and matched benign tissues reflect the genetic basis of tumorigenesis ${ }^{[28,29]}$; and (2) genes differentially expressed between low and high Gleason scores correlate with tumor aggressiveness ${ }^{[30]}$. Therefore, in addition to being used as prognostic markers, the iPAM genes selected from those DEGs are likely to be functionally relevant to cancer progression. From Ingenuity Pathway Analysis (IPA), those DEGs were enriched in pathways of immune response, cell adhesion, and degradation of extracellular matrix [Supplementary Table 5]. Enrichment of pathways in immune response was among the up-regulated DEGs identified only in the young group (highlighted pathways in Supplementary Table 5). Estimation of abundance of immune cells in tumor and benign tissues from $\mathrm{COH}$ patients corroborated a role of more pronounced immune responses to tumor development in young patients than in old patients [Supplementary Table 2 and Figure 4A]. Ten of 36 iPAM genes were linked to immune-related pathways [Table 3] and showed larger metastasis-associated differences (FDR $<0.10$, Supplementary Table 6 in the young group than in the old group from Decipher GRID samples [Figure $2 \mathrm{C}$ and Figure $2 \mathrm{D}$ and Supplementary Figure 4]. Furthermore, the estimated abundance of immune cells in primary tumor samples from the five Decipher GRID data sets was positively associated with the development of metastasis in young and middle-aged patients with no significant association in old patients [Supplementary Table 3 and Figure $4 \mathrm{~B}$ ]. The positive correlation between the immune scores (the abundance of immune cell types) and 
the risk scores of metastasis generated by the iPAM classifier for validation samples was highly significant $\left(P\right.$-value $\left.=2.68 \times 10^{-11}\right)$. Accuracy of early metastasis prediction from the iPAM classifier was higher for young patients $(\mathrm{AUC}=0.87)$ and middle-aged patients $(\mathrm{AUC}=0.82)$ than for old patients $(\mathrm{AUC}=0.69)$. The trend of differences in prediction accuracy among the age groups is consistent with the observation that the metastasis-associated immune responses in the tumor microenvironment in young and middleage patients were more pronounced than in old patients. Taken together, these findings show that more pronounced metastasis-associated immune responses in tumor microenvironment are found in young and middle-age patients than in old patients, potentially explaining the difference in accuracy of metastasis prediction among the three age groups.

This study has limitations. Although the sample sets included 58 young patients ( $\leq 50$ years) for identifying DEGs and 197 young patients ( $\leq 55$ years) for iPAM classifier development and validation, the sample size of young patients is modest. Second, we identified genes that serve as prognostic biomarkers and also show functional relevance to cancer progression based on IPA analysis; however, performing functional studies on the role of the 36 iPAM genes on cancer progression was beyond the scope of this study. Moreover, as previously reported, the Decipher GRID data sets may over-represent patients with adverse clinicopathologic features ${ }^{[31]}$.

We identified an iPAM classifier for prediction of early metastasis; the prediction accuracy of the iPAM classifier was higher for young ( $\leq 55$ years) and middle-aged patients (56-70 years) than for old patients (> 70 years). We also provided evidence that this age-related difference in prediction accuracy can be explained by differential immune responses to metastasis development among the three age groups.

\section{DECLARATIONS}

\section{Acknowledgements}

The authors thank Charles Warden for downloading the decipher GRID data sets from the Decipher Biosciences.

\section{Authors' Contributions}

Conception and design: Neuhausen SL, Ding YC

Development of methodology: Neuhausen SL, Ding YC

Acquisition of data: Wu H, Davicioni E, Karnes RJ, Klein EA, Den RB

Analysis and interpretation of data: Neuhausen SL, Ding YC

Writing, review, and/or revision of the manuscript: Neuhausen SL, Ding YC

Administrative, technical, or material support: Steele L

Study supervision: Neuhausen SL

\section{Availability of data and materials}

Data will be available upon request.

\section{Financial support}

This project was supported by the Morris and Horowitz Families Professorship (SLN). Research reported in this publication included work performed in the Integrative Genomics and Pathology, Cores supported by the National Cancer Institute of the National Institutes of Health under award number P30CA033572. The content is solely the responsibility of the authors and does not necessarily represent the official views of the National Institutes of Health.

\section{Conflicts of interest}

All authors declared that there are no conflicts of interest. 


\section{Ethical approval and consent to participate}

This study was approved by the City of Hope (COH) Institutional Review Board (IRB07244).

\section{Consent for publication}

Documents of consent for publication are available if requested.

\section{Copyright}

(c) The Author(s) 2021.

\section{REFERENCES}

1. Howlader N, Noone AM, Krapcho M, et al. SEER Cancer Statistics Review, 1975-2017. National Cancer Institute. Bethesda, MD. 2019, https://seer.cancer.gov/csr/1975_2017/, November 2019 SEER data submission(posted to the SEER web site, April 2020.

2. Li J, German R, King J, et al. Recent trends in prostate cancer testing and incidence among men under age of 50. Cancer Epidemiol 2012;36:122-7.

3. Bleyer A, Spreafico F, Barr R. Prostate cancer in young men: An emerging young adult and older adolescent challenge. Cancer 2020;126:46-57.

4. Kucera R, Pecen L, Topolcan O, et al. Prostate cancer management: long-term beliefs, epidemic developments in the early twenty-first century and 3PM dimensional solutions. EPMA J 2020;11:399-418.

5. Kinnear NJ, Kichenadasse G, Plagakis S, et al. Prostate cancer in men aged less than 50 years at diagnosis. World J Urol 2016;34:1533-9.

6. Mahal AR, Butler S, Franco I, et al. Conservative management of low-risk prostate cancer among young versus older men in the United States: Trends and outcomes from a novel national database. Cancer 2019;125:3338-46.

7. Bill-Axelson A, Holmberg L, Garmo H, et al. Radical Prostatectomy or Watchful Waiting in Prostate Cancer - 29-Year Follow-up. N Engl J Med 2018;379:2319-29.

8. Boström PJ, Bjartell AS, Catto JW, et al. Genomic Predictors of Outcome in Prostate Cancer. Eur Urol 2015;68:1033-44.

9. Ding Y, Wu H, Warden C, et al. Gene Expression Differences in Prostate Cancers between Young and Old Men. PLoS Genet 2016;12:e1006477.

10. Salinas CA, Tsodikov A, Ishak-Howard M, Cooney KA. Prostate cancer in young men: an important clinical entity. Nat Rev Urol 2014;11:317-23.

11. Steurer S, Mayer PS, Adam M, et al. TMPRSS2-ERG fusions are strongly linked to young patient age in low-grade prostate cancer. Eur Urol 2014;66:978-81.

12. Weischenfeldt J, Simon R, Feuerbach L, et al. Integrative genomic analyses reveal an androgen-driven somatic alteration landscape in early-onset prostate cancer. Cancer Cell 2013;23:159-70.

13. Zhao SG, Jackson WC, Kothari V, et al. High-throughput transcriptomic analysis nominates proteasomal genes as age-specific biomarkers and therapeutic targets in prostate cancer. Prostate Cancer Prostatic Dis 2015;18:229-36.

14. Smyth GK, Michaud J, Scott HS. Use of within-array replicate spots for assessing differential expression in microarray experiments. Bioinformatics 2005;21:2067-75.

15. Erho N, Crisan A, Vergara IA, et al. Discovery and validation of a prostate cancer genomic classifier that predicts early metastasis following radical prostatectomy. PLoS One 2013;8:e66855.

16. Tibshirani R, Hastie T, Narasimhan B, Chu G. Diagnosis of multiple cancer types by shrunken centroids of gene expression. Proc Natl Acad Sci U S A 2002;99:6567-72.

17. Wang S, Zhu J. Improved centroids estimation for the nearest shrunken centroid classifier. Bioinformatics 2007;23:972-9.

18. Blagus R, Lusa L. Improved shrunken centroid classifiers for high-dimensional class-imbalanced data. BMC Bioinformatics 2013;14:64.

19. Karnes RJ, Bergstralh EJ, Davicioni E, et al. Validation of a genomic classifier that predicts metastasis following radical prostatectomy in an at risk patient population. $J$ Urol 2013;190:2047-53.

20. Klein EA, Yousefi K, Haddad Z, et al. A genomic classifier improves prediction of metastatic disease within 5 years after surgery in nodenegative high-risk prostate cancer patients managed by radical prostatectomy without adjuvant therapy. Eur Urol 2015;67:778-86.

21. Den RB, Feng FY, Showalter TN, et al. Genomic prostate cancer classifier predicts biochemical failure and metastases in patients after postoperative radiation therapy. Int J Radiat Oncol Biol Phys 2014;89:1038-46.

22. Aran D, Hu Z, Butte AJ. xCell: digitally portraying the tissue cellular heterogeneity landscape. Genome Biol 2017;18:220.

23. Wilcox R. Introduction to Robust Estimation and Hypothesis Testing. 4th ed. Academic press; 2016.

24. Jegerlehner S, Chiolero A, Aujesky D, et al; NICER Working Group. Recent incidence and surgery trends for prostate cancer: Towards an attenuation of overdiagnosis and overtreatment? PLoS One 2019;14:e210434.

25. Loeb S, Bjurlin MA, Nicholson J, et al. Overdiagnosis and overtreatment of prostate cancer. Eur Urol 2014;65:1046-55.

26. Klein EA, Cooperberg MR, Magi-Galluzzi C, et al. A 17-gene assay to predict prostate cancer aggressiveness in the context of Gleason grade heterogeneity, tumor multifocality, and biopsy undersampling. Eur Urol 2014;66:550-60.

27. Cuzick J, Swanson GP, Fisher G, et al. Prognostic value of an RNA expression signature derived from cell cycle proliferation genes in patients with prostate cancer: a retrospective study. The Lancet Oncology 2011;12:245-55.

28. Murphy LC, Simon SL, Parkes A, et al. Altered expression of estrogen receptor coregulators during human breast tumorigenesis. Cancer 
Res 2000;60:6266-71.

29. Kho AT, Zhao Q, Cai Z, et al. Conserved mechanisms across development and tumorigenesis revealed by a mouse development perspective of human cancers. Genes Dev 2004;18:629-40.

30. Humphrey PA. Gleason grading and prognostic factors in carcinoma of the prostate. Mod Pathol 2004;17:292-306.

31. Spratt DE, Yousefi K, Deheshi S, et al. Individual Patient-Level Meta-Analysis of the Performance of the Decipher Genomic Classifier in High-Risk Men After Prostatectomy to Predict Development of Metastatic Disease. J Clin Oncol 2017;35:1991-8. 